\title{
Application of Accurate Angle of Healthy Side Upper Limb in Body Posture in Radical Mastectomy
}

\author{
Lian Huizhao ${ }^{1}$, Guo Xiaoxia ${ }^{2, ~}$, Rao Jingmin ${ }^{2}$, Qian Yuxiu ${ }^{1}$, Hou Lihuan ${ }^{1}$, Xiao Xiaolu ${ }^{1}$ \\ ${ }^{1}$ Department of Operating Room, The First Affiliated Hospital of Jinan University, Guangzhou, China \\ ${ }^{2}$ The First Affiliated Hospital, Jinan University, Guangzhou, China
}

Email address:

guoxiaoxia2019@sina.com (Guo Xiaoxia)

${ }^{*}$ Corresponding author

\section{To cite this article:}

Lian Huizhao, Guo Xiaoxia, Rao Jingmin, Qian Yuxiu, Hou Lihuan, Xiao Xiaolu. Application of Accurate Angle of Healthy Side Upper Limb in Body Posture in Radical Mastectomy. International Journal of Clinical and Experimental Medical Sciences. Vol. 6, No. 1, 2020, pp. 12-16. doi: $10.11648 /$ j.ijcems.20200601.13

Received: February 20, 2020; Accepted: March 2, 2020; Published: March 10, 2020

\begin{abstract}
Objective: We attempt to explore the effect of accurate angle of healthy side upper limb on body posture in radical mastectomy. Methods: We enrolled 150 patients who received radical mastectomy in our hospital and averagely randomized them into three groups: control group A, control group B and observation group. In control group A, the intraoperative abduction angle of healthy side upper limb was $90^{\circ}$, in control group B it was $0^{\circ}$, and observation group $75-80^{\circ}$. We scored the comfort level of the healthy side upper limb of patients in three groups prior to anesthesia induction and 24 hours after operation. At the end of operation, we scored the first assistant surgeon's, anesthetist's and circulating nurse's satisfaction with standing space, the convenience of intravenous administration and convenience of observing transfusion during operation respectively. Results: There was significant difference in the comfort level between observation group and control group A, B $(P<0.05)$. The first assistant surgeon's satisfaction with standing space was $100 \%$ in all three groups with no significant difference $(P>0.05)$. Anesthetist's satisfaction with the convenience of intravenous administration during operation was $100 \%$ in observation group and control group A while it was $36 \%$ in control group B. There was a significant difference $(P<0.05)$. Circulating nurse's satisfaction with the convenience of observing transfusion during operation was $100 \%$ in observation group and control group A while it was $30 \%$ in control group B. There was a significant difference $(P<0.05)$. Conclusions: When the accurate angle of healthy side upper limb in the body posture in radical mastectomy is $75-80^{\circ}$, it does not affect the standing space of the first assistant surgeon, intraoperative administration by anesthetist and observation of transfusion by circulating nurse, and avoids intraoperative position-related complications of patients.
\end{abstract}

Keywords: Accuracy, Healthy Side Upper Limb, Angle, Radical Mastectomy

\section{Introduction}

It is an important task to arrange the body posture of patients before operation. Correct body posture of operation ensures good exposure of operating field, facilitating doctor's operation, and at the same time ensures comfort level as much as possible so as to reduce impact on patients' blood pressure, breath and heart rate, etc. Medical staff should avoid damage to nerves or limbs due to long-time pressure on a certain body part and even bedsore caused by inappropriate body posture. In addition, they should ensure comfort level and safety of patients to avoid position-induced complications [1-3].
Supine position is the commonly assumed body posture for radical mastectomy. In this position, the two upper limbs are extended horizontally. Abduction of affected upper limb exposes the operating field fully and allows the limb to be fixed to the limb rack of the operating table. It should be noticed that the limbs should not be extended too much to avoid brachial plexus paralysis. In addition, soft pillow should be put under the back to elevate patient's axilla so that the operator can isolate the adipose tissue around blood vessels and nerves. So far, Guide to Operating Room Nursing Practice [4, 5] suggests that the abduction angle of upper limbs should be no more than 90 degrees $\left(\leq 90^{\circ}\right)$ when assuming supine position to avoid damage to brachial plexus. However, the guide does not make 
the best angle clear, and mentions very little about the angle of healthy side upper limb. It remains unclear whether deciding the accurate angle of the healthy side upper limb can avoid or reduce discomfort of the healthy upper limb after operation. We randomized 150 patients who received radical mastectomy in our hospital from June 2018 to December 2019 into three groups and arrange patients of different groups to assume different angles of the healthy side upper limb. We found that an accurate angle of the healthy side upper limb can avoid or reduce occurrence of discomfort in the healthy upper limb after operation. The research is reported as follows.

\section{Data and Methods}

\subsection{General Data}

We enrolled 150 female patients who received radical mastectomy in our hospital from June 2018 to December 2019 and averagely randomized them into three groups. In the control group A, patients age from 30 to 58 with an average age of $44.46 \pm 7.09$, and there are 18 cases of modified radical mastectomy, 23 cases of total mastectomy and sentinel lymph node biopsy, and 9 cases of breast reservation radical correction. Among all the cases, 28 are for left breast and 22 for right breast. In the control group B, patients age from 31 to 56 with an average age of $44.52 \pm 6.80$, and there are 15 cases of modified radical mastectomy, 20 cases of total mastectomy and sentinel lymph node biopsy, and 15 cases of breast reservation radical correction, among which 24 cases are for left breast and 26 cases for right breast. In the observation group, patients age from 32 to 59 with an average of $43.62 \pm 7.04$, and there are 23 cases of modified radical mastectomy, 17 cases of total mastectomy and sentinel lymph node biopsy, and 10 cases of breast reservation radical correction. Among all 50 cases, 22 cases are for left breast and 28 for right breast. There is no significant difference in the sex, age and operational methods between three groups $(P>0.05)$.

The inclusion criteria are: the pathology results of the patients must show cancer in only one breast and patients possess ability to communicating. The operation takes $3-3.5 \mathrm{~h}$ and the infusion is through the healthy side upper limb. Exclusion criteria include: the breast cancer patients are males or the patients have cancer in two breasts. The breast cancer patients have received operation of cancer for one breast. Patients have history of periarthritis of shoulder on the healthy side or cervical spondylosis. Patients have insufficiency of healthy side upper limb or intraoperative infusion is through deep vein.

\subsection{Methods}

\subsubsection{Positioning of Healthy Side Upper Limb}

Positioning of the healthy side upper limb of patients in three groups were conducted by two professional nurses and electric Mindray operating bed and the same adjustable upper limb holder were adopted. In radical mastectomy, the abduction angle of the healthy side upper limb of patients in control group A was $90^{\circ}$ : the patient lied on the back on the operating bed, and aligned the top of the head with the edge of the bed, and the posterior axillary line of the affected side with the bedside edge. After the patient was anesthetized, a gel axillary pad was placed on the affected side to elevate the axilla so as to fully expose it. The upper limb of the affected side was abducted and put on the hand holder. A rectangular pillow was placed on the hand holder to elevate the upper limb to the same height as the body. The abduction angle of the upper limb of the healthy side was $90^{\circ}$, and a venous channel was established on the back of the hand, with the palm side facing down to prevent the vein from being compressed which might affect the venous return. The distal joint was slightly higher than the proximal joint. The restraint band fixed the healthy side upper limb. A popliteal pad was placed at the popliteal space of both lower limbs, and a heel pad under the feet. A restraint band was used to fix at $5 \mathrm{~cm}$ above the knee joint with space left to accommodate one finger [6]. After routine surgical disinfection, the operator sat below the upper limb of the affected side, and the first assistant stood below the upper limb of the healthy side. In the control group B, the abduction angle of the healthy side upper limb of patients was $0^{\circ}$ : the healthy side upper limb was put along the body and fixed with bed sheet. The other procedures were the same as those of control group A. In the observation group, the abduction angle of healthy side upper limb was $75-80^{\circ}$ : we used a goniometer to measure the angle between $75-80^{\circ}$, and fixed the upper arm holder at the point, and then placed the upper limb of the patient's healthy side on the holder. The other procedures were the same as those of control group A and B.

\subsubsection{Outcome Measurements}

i. Comfort Level of Healthy Side Upper Limb of Patients

Assessment time: we assessed the comfort level of the healthy side upper limb before anesthesia induction and 24 hours after operation.

Assessment method: there were four grades for the comfort level of patients' body and arms and legs prior to operation: 0 indicates no discomfort; 1 means mild and bearable discomfort or ache; 2 means moderate discomfort and obvious ache in body or limbs, but the ache can be relieved after change of body posture and physical therapy; 3 indicates severe discomfort and severe ache in body or limbs and the ache cannot be relieved after change of body posture and physical therapy [7].

ii. Satisfaction of Related Medical Staff

At the end of the operation, we assessed the first assistant surgeon's satisfaction with standing space, circulating nurse's satisfaction with convenience of observing venipuncture site and anesthetist's satisfaction with convenience of intraoperative administration. The results included "satisfied" and "dissatisfied".

\subsection{Statistical Methods}

Statistical analysis of all data was done with the statistical software SPSS20.0. We used $\chi^{2}$ test to analyze the relationship of accurate angle of healthy side upper limb in radical mastectomy for breast cancer between $75-80^{\circ}$ and $90^{\circ}, 0^{\circ}$. And $P<0.05$ indicates significant difference. 


\section{Results}

\subsection{Comparison of Comfort Level of Healthy Side Upper Limb of Patients}

Before anesthesia induction and 24 hours after operation, we compared the comfort level of healthy side upper limb of patients having received radical mastectomy between the three groups. There was significant difference between observation group and control group $\mathrm{A}$ and $\mathrm{B}(P<0.05)$ as shown in the Table 1.

Table 1. Comparison of Comfort Level of Healthy Side Upper Limb between Three Groups (case).

\begin{tabular}{|c|c|c|c|c|c|c|c|c|c|}
\hline \multirow{2}{*}{ Groups } & \multirow{2}{*}{ Cases } & \multicolumn{4}{|c|}{ Comfort level before anesthesia induction } & \multicolumn{4}{|c|}{ Comfort level 24 hours after operation } \\
\hline & & $\mathbf{0}$ & 1 & 2 & 3 & $\mathbf{0}$ & 1 & 2 & 3 \\
\hline Control group A & 50 & 35 & 15 & 0 & 0 & 28 & 20 & 3 & 0 \\
\hline Observation group & 50 & 46 & 4 & 0 & 0 & 43 & 7 & 0 & 0 \\
\hline$\chi^{2}$ & & 7.78 & & & & 13.38 & & & \\
\hline$P$ & & 0.02 & & & & 0.01 & & & \\
\hline
\end{tabular}

\subsection{Comparison of Satisfaction of Related Medical Staff During Operation}

There was no significant difference in the first assistant surgeon's satisfaction with standing space between observation group and control group $\mathrm{A}$ and $\mathrm{B}(P>0.05)$. In observation group and control group A, anesthetist's satisfaction with the convenience of intraoperative venous administration was $100 \%$ while in control group $\mathrm{B}$, it was $36 \%$. There was a significant difference. In observation group and control group A, circulating nurse's satisfaction with the convenience of observing infusion site was $100 \%$ while in control group B, it was $30 \%$. There was a significant difference (as shown in the Table 2).

Table 2. Comparison of Satisfaction of Related Medical Staff during Operation (\%).

\begin{tabular}{|c|c|c|c|c|c|c|c|c|c|c|}
\hline \multirow{2}{*}{ Groups } & \multirow{2}{*}{$\mathbf{N}$} & \multicolumn{3}{|c|}{ Standing space } & \multicolumn{3}{|c|}{$\begin{array}{l}\text { Convenience of venous } \\
\text { administration }\end{array}$} & \multicolumn{3}{|c|}{$\begin{array}{l}\text { Convenience of observing } \\
\text { venipuncture site }\end{array}$} \\
\hline & & $\begin{array}{l}\text { Very } \\
\text { satisfied }\end{array}$ & satisfied & dissatisfied & $\begin{array}{l}\text { Very } \\
\text { satisfied }\end{array}$ & satisfied & dissatisfied & $\begin{array}{l}\text { Very } \\
\text { satisfied }\end{array}$ & satisfied & dissatisfied \\
\hline Control group A & 50 & 30 & 20 & 0 & 40 & 10 & 0 & 46 & 4 & 0 \\
\hline Control group B & 50 & 32 & 16 & 0 & 0 & 18 & 32 & 0 & 15 & 35 \\
\hline Observation group & 50 & 35 & 15 & 0 & 45 & 5 & 0 & 44 & 6 & 0 \\
\hline$P$ & & 0.57 & & & 0.00 & & & 0.00 & & \\
\hline
\end{tabular}

\section{Discussion}

\subsection{Comparison of Different Angles of Healthy Side Upper Limb in Radical Mastectomy}

Guide to Operating Room Nursing Practice suggests the abduction angle of upper limbs should be no more than 90 degrees $\left(\leq 90^{\circ}\right)$ in supine position to avoid brachial plexus injury. A clear operating field requires abduction of limb on affected side and the most common abduction angle is $90^{\circ}$. However, the guide does not make clear suggestion about the position angle of the healthy side upper limb. There are usually two common angles: $90^{\circ}$ (the healthy side upper limb is abducted as the affected upper limb) and $0^{\circ}$ (the healthy side upper limb is put along the body) [4, 8-10]. Generally, radical mastectomy takes $3-3.5 \mathrm{~h}$. The abduction angles of $90^{\circ}$ and $0^{\circ}$ are not compulsive positions while position-related complications will increase with increase in the duration of the operation. For patients with a healthy side upper limb angle of $0^{\circ}$, in order to avoid electric burns of finger or palm caused by upper limb displacement during surgery, the healthy upper limbs are usually wrapped on a cloth sheet next to the body.
Patients are unable to perceive and tell comfort level after general anesthesia $[11,12]$. On the other hand, when clearing patients' axillary lymph nodes, the surgeon, in order for a fuller explosion of the operating field, will turn the operating bed to the healthy side by about $10^{\circ}$ which causes the healthy side upper limb to be compressed and complications induced by brachial plexus injury. Therefore, the healthy side upper limb abduction angles of $90^{\circ}$ and $0^{\circ}$ are of certain disadvantages.

\subsection{To Determine an Accurate Angle of the Healthy Side Upper Limb to Improve Patients' Comfort Level}

Supine position is the common body posture in radical mastectomy. During the operation, the regular abduction angle of healthy side upper limb is $\leq 90^{\circ}$ or $0^{\circ}$ in order to provide enough standing space for the first assistant surgeon. However, mechanical positioning of the healthy side upper limb ignores the comfort level of patients and nurses' humanistic care for patients [13]. The current research, based on the Guide to Operating Room Nursing Practice, explores the best abduction angle of healthy side upper limb between $\leq$ $90^{\circ}$ and $0^{\circ}$ when assuming supine position in radical mastectomy. We adjust the optimal angle of the upper limb on 
the healthy side of the patient according to patient's feeling. The patient's best comfort state is that the angle of the upper limb of the healthy side is $75-80^{\circ}$. In this research, the proportion of healthy side upper limb of comfort level 0 in the observation group was $86 \%-92 \%$, in the control group A was $15.6 \%-70 \%$, and in the control group B was $64 \%-80 \%$. According to the comfort level, it is advised to set the angle of the healthy side upper limb as $75-80^{\circ}$ during the radical mastectomy.

\subsection{No Impact of Accurate Angle of Healthy Side Upper Limb on the Convenience of Non-operative Practice}

Patients in this research need to be infused on the healthy side upper limb during the operation for intraoperative administration by anesthetists and observing venipuncture site by circulating nurses. When the angle of the upper limb on the healthy side is $90^{\circ}$ or $75-80^{\circ}$, the patient's upper limb is abducted, and the infusion site is far away from the operating bed and above the side of the first assistant surgeon. The anesthetist's administration and circulating nurse's observation of the puncture site have no interference on the first assistant surgeon. When the angle of the healthy side upper limb is $0^{\circ}$, the healthy upper limb is commonly wrapped along the body with bed sheet. When the anesthetist approaches the operating bed for administration or circulating nurse for observing the infusion site, both of them need to loosen the bed sheet and will take up the space of the first assistant surgeon, disturbing the first assistant surgeon. On the other hand, when cleaning the patient's axillary lymph nodes, the operator in order to better expose the operating field, will turn the operating bed to the healthy side by about $10^{\circ}$. As a result, the healthy upper limb will be compressed which affects the speed of infusion during the operation. Surveys on the satisfaction of related medical staff in the three groups show that: the first assistant surgeon's satisfaction with the standing space is $100 \%$; the anesthetist's satisfaction with the convenience of intraoperative administration is $100 \%$ in control group A and observation group while it is $36 \%$ in control group B; and the circulating nurse's satisfaction with the convenience of observing the infusion site is $100 \%$ in both the control group A and the observation group while it is $36 \%$ in control group B. The results are consistent with related research [14-15].

\section{Conclusions}

In conclusion, the accurate angle of healthy side upper limb $75-80^{\circ}$ based on the principles of routine postural placement in surgical nursing does not affect the standing space of the first assistant surgeon, intraoperative administration by anesthetist and observing the infusion site by circulating nurse, and avoiding brachial plexus paralysis and discomfort of the healthy upper limb after operation. What's more, the angle also avoids position-related complications and improves patients' comfort level, embodying nurse's respect for the patients and patients' trust for the nurse. Therefore, this angle is worth application in the positioning of the two upper limbs when assuming supine position during operation.

\section{References}

[1] Kamel, I., \& Barnette, R. (2014). Positioning patients for spine surgery: avoiding uncommon position-related complications. World Journal of Orthopaedics, 5 (4), 425-443.

[2] Uppal, R. S., Casaer, B., Landuyt, K. V., \& Blondeel, P. (2009). The efficacy of preoperative mapping of perforators in reducing operative times and complications in perforator flap breast reconstruction. Journal of Plastic Reconstructive \& Aesthetic Surgery, 62 (7), 859-864.

[3] Tuija Leinonen, \& Helena Leino-Kilpi. (1999). Research in peri-operative nursing care. Journal of Clinical Nursing, 8 (2), 123-138.

[4] N Al-Yateem, M Al-Tamimi, Brenner, H Altawil, \& S Brownie. (2018). Research priorities for specialized nursing practice in the united arab emirates. International Nursing Review, 65 (3), 381-391.

[5] Kasagi, Y., Okutani, R., \& Oda, Y. (2015). Specialized operating room for cesarean section in the perinatal care unit: a review of the opening process and operating room management. Journal of Anesthesia, 29 (1), 149-151.

[6] Yue Guo, Yan Li, Kuaile Zhao, Xiao Yue, \& Tiyu Zhao. (2017) Effects of curvilinear supine position on tissue interface pressure: a prospective before-and-after study. Journal of Wocn, 44 (5), 450-454.

[7] Flaherty, Geraldine G., \& Fitzpatrick, Joyce J. (1978).. Relaxation technique to increase comfort level of postoperative patients: a preliminary study. Nursing Research, 27 (6), 352-355.

[8] Lorry Schoenly. (2015). Research priorities in correctional nursing practice: results of a three-round delphi study. Journal of Correctional Health Care, 21 (4), 400-7.

[9] Mcgarvey, H. E., Chambers, M. G. A., \& Boore, J. R. P. (2000) Development and definition of the role of the operating department nurse: a review. Journal of Advanced Nursing, 32 (5), 1092-1100.

[10] Ann-Catrin Blomberg, Lindwall, L., \& Bisholt, B. (2019). Operating theatre nurses' self-reported clinical competence in perioperative nursing: a mixed method study. Nursing Open, 6 (4), 1510-1518.

[11] Woernle, C. M., Sarnthein, J., Foit, N. A., \& Krayenbühl, Niklaus. (2013). Enhanced serum creatine kinase after neurosurgery in lateral position and intraoperative neurophysiological monitoring. Clinical Neurology and Neurosurgery, 115 (3), 266-269.

[12] Neidert, M. C., Losa, M., Regli, L., \& Sarnthein, J. (2015). Elevated serum creatine kinase after neurosurgeries in lateral position with intraoperative neurophysiological monitoring is associated with op duration, bmi and age. Clinical Neurophysiology, 126 (10), S138824571500005X.

[13] Vassileios, Karampelias, Dimitrios, Koukouras, Evaggelos, \& Tzorakoleftherakis, et al. (2019). Breast cancer section analysis correlates with sentinel lymph node biopsies: precision and topographic anatomy. Breast disease, 38 (1), 1-5. 
[14] Andrea Michalski, John Atyeo, Jennifer Cox, \& Marianne Rinks. (2012). Inter- and intra-fraction motion during radiation therapy to the whole breast in the supine position: a systematic review. Journal of Medical Imaging \& Radiation Oncology, 56 (5), 499-509.
[15] Lillie, H. M., Venetis, M. K., \& Chernichky-Karcher, S. M.. (2018). "He would never let me just give up": communicatively constructing dyadic resilience in the experience of breast cancer. Health Communication, 33 (12): 1516-1524. 